\title{
Effect of Frequency and Number of Piezoelectric Probes in Sonication- assisted Exfoliation of Graphite Layers into Graphene Oxide
}

\author{
Aminah Nur Aisyah, ${ }^{1}$ Achmad Ainul Fikri, ${ }^{1}$ Suhufa Alfarisa, ${ }^{2}$ Agus Purwanto, ${ }^{3}$ \\ Sumarna $^{3}$ and Wipsar Sunu Brams Dwandaru ${ }^{{ }^{*}}$ \\ ${ }^{1}$ Colloids and Soft Material Group, Physics Education Department, \\ Faculty of Mathematics and Natural Science, Universitas Negeri Yogyakarta, \\ Karangmalang Complex, Yogyakarta 55281, Indonesia \\ ${ }^{2}$ Department of Physics, Universitas PGRI Palembang, Palembang 30251, Indonesia \\ ${ }^{3}$ Signal Processing Group, Physics Education Department, \\ Faculty Mathematics and Natural Science, Universitas Negeri Yogyakarta, \\ Karangmalang Complex, Yogyakarta 55281, Indonesia \\ ${ }^{*}$ Corresponding author: wipsarian@uny.ac.id
}

Published online: 25 August 2018

To cite this article: Aisyah, A. N. et al. (2018). Effect of frequency and number of piezoelectric probes in sonication-assisted exfoliation of graphite layers into graphene oxide. J. Phys. Sci., 29(2), 121-135, https://doi.org/10.21315/jps2018.29.2.8

To link to this article: https://doi.org/10.21315/jps2018.29.2.8

\begin{abstract}
A self-custom-made ultrasound generator using piezoelectric probes has been constructed to reduce graphite layers via liquid-phase exfoliation into graphene oxide (GO) material. The ultrasound frequency and the number of piezoelectric probes are varied with values of $20 \mathrm{kHz}, 30 \mathrm{kHz}$ and $35 \mathrm{kHz}$, and 1 probe, 2 probes and 3 probes, respectively. The solutions obtained from the sonication process show a temperature increase of $1^{\circ} \mathrm{C}$ or $2^{\circ} \mathrm{C}$ compared to room temperature. Colour changes of the solution before sonication, after sonication, and after being left overnight are also exhibited; that is from dark, dark blue, to greyish blue, respectively. The solutions are then characterised using UV-Vis spectrophotometer and scanning electron microscope (SEM). The UV-Vis results show the presence of GO material at an absorbance peak of $270 \mathrm{~nm}$. Increasing the frequency and number of the probes decreases the absorbance peaks of the solutions. The best GO sample solution is obtained for an ultrasound frequency of $35 \mathrm{kHz}$ and using 3 piezoelectric probes. Images from SEM show rod-like carbon materials stacked on top of each other in the form of flower-like structures. The widths of these rod-like materials vary from 1 to 2 microns, whereas the thicknesses of these materials are around $300 \mathrm{~nm}$ to 1.5 microns.
\end{abstract}

Keywords: Piezoelectric, self-custom-made ultrasound generator, graphene oxide, liquidphase exfoliation, exfoliation of graphite 


\section{INTRODUCTION}

A piezoelectric is an electronic device that may serve as a sensor or even a speaker for audible sound and ultrasound waves by exploiting the electric charges generated on certain solid materials as a result of mechanical pressure. The device has been utilised for various everyday electrical appliances such as engine and pressure sensors, sonar equipment, ultrasonic cleaning, ultrasound imaging printers and so forth. ${ }^{1-5}$ Piezoelectric is still a subject of advanced studies, including piezoelectric nanogenerator, vanadium $\mathrm{ZnO}(\mathrm{VZO})$ and lead-free $0.5\left[\mathrm{Ba}_{0.7} \mathrm{Ca}_{0.3} \mathrm{TiO}_{3}\right]-$ $0.5\left[\mathrm{Ba}\left(\mathrm{Zr}_{0.2} \mathrm{Ti}_{0.8}\right) \mathrm{O}_{3}\right]$ (BCZT) ceramics thin films piezoelectric, omnidirectional shear horizontal piezoelectric transducer, and so on. ${ }^{6-9}$ One method to produce this device is by assembling the piezoelectric actuators to generate vibration responses with various high-amplitude modes. ${ }^{10}$ In this study, piezoelectricity is used as an ultrasound speaker incorporated into a self-custom-made ultrasound generator. The ultrasound generator is ultimately constructed to reduce the layers of graphite materials into graphene oxide (GO) materials. This is conducted by exposing high frequency ultrasound waves into graphite materials in a liquid phase. The high frequency oscillations disrupt the van der Waals bond between adjacent graphite layers such that they become exfoliated into thinner layers of GO.

The synthesis of GO based on liquid exfoliation (LE) using high frequency sound waves, a kitchen blender and electrolysis is already established. ${ }^{11-17}$ In fact, other excellent methods in producing GO on a large scale are also acknowledged, such as Hummers method, chemical vapour deposition (CVD), epitaxial growth, reduction of $\mathrm{GO}(\mathrm{rGO})$, mechanical exfoliation $(\mathrm{ME})$, and recently there is also a fluid dynamic route. ${ }^{18-28}$ Here, we use piezoelectric as it is easy to obtain and economically inexpensive. Furthermore, the construction of the self-custom-made ultrasound generator is directed to give an alternative option amongst the diverse literatures of GO production. This option comprises a simple assembly and usage of the ultrasound generator, minimum cost comparable to standard sonicator, and potential ability to produce large scale GO. The present study is a significant development of a previous study with newly modified construction of the selfcustom-made ultrasound generator. ${ }^{29}$ Moreover, the frequency and the number of piezoelectric probes of the ultrasound generator are varied. The objective of this study is to determine the effect of the aforementioned variables towards the exfoliation of graphite layers into GO materials based on UV-Visual (UV-Vis) spectrophotometer and SEM results. To the knowledge of the authors, this has not been reported before.

Carbon powder from Faber-Castell 2B pencils is used as a source of graphite. The powder is made into a solution consisting of surfactant contained in household detergent. The detergent is added to assist the exfoliation process. ${ }^{30}$ The addition 
of surfactant may generate defects upon the GO produced, but the material remains stable and in good quality. ${ }^{31,32}$

GO is a precursor in obtaining graphene. The latter is a highly sought material with outstanding physical and chemical properties such as high Young's modulus, excellent optical transparency, good thermal conductivity, high electrical conductivity and high electron conductivity. ${ }^{33}$ Nonetheless, GO itself is vastly studied for its variety of applications, including bio-sensor, hydrogen storage, filtration membranes, biomedical and optical materials, and anti-bacterial added with silver nanoparticles and bacterial cellulose. ${ }^{34-45}$ Understanding the properties of GO and exploring its promising applications have led to a great increase of research worldwide.

\section{THE SELF-CUSTOM-MADE ULTRASOUND GENERATOR}

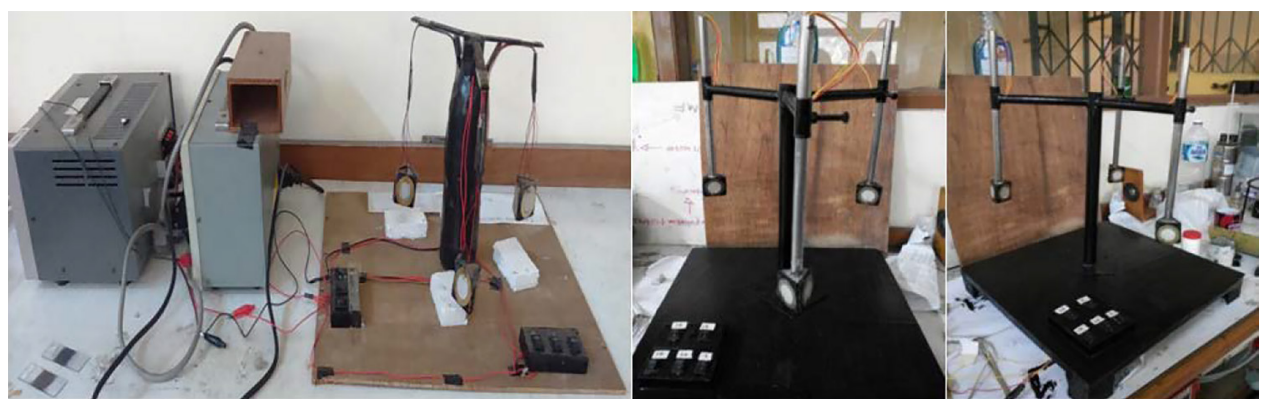

Figure 1: The initial construction of the self-custom-made ultrasound generator (leftmost photo) and a modification made to the piezoelectric probe installation (middle and right-most photos).

The main device used in this study is a self-custom-made ultrasound generator, which may be observed in Figure 1. The initial construction of the ultrasound generator may be viewed in the left-most picture of Figure 1. ${ }^{29}$ The apparatus needed to assemble the ultrasound generator are, from left to right of the left-most picture of Figure 1: (1) an audio generator (CSi/SPECO SS-1); (2) an amplifier (Uchida TA-2MS); and (3) an installation of piezoelectric probes. The latter installation consists of three probe assemblies, each containing three piezoelectric probes hanging freely on flexible wires span by thin wooden sticks mounted on top of a support made from an empty used-drinking water bottle wrapped with black duct tape. The triangular shaped assembly of the probes is created in order to expose ultrasound in all directions of space while minimising the number of probes used in each arrangement. 
A modification is made to the probe assembly in order to improve its physical feature including making it more rigid. The modification may be observed in the middle and right-most pictures of Figure 1. The main support is made of metal with three arms reaching out at the top. A hole is located at the end of each arm, equipped with a screw to tighten the hole. The probes are placed at the end of a long metal cylinder with a diameter that may fit into the aforementioned hole. The wiring is put inside the long metal cylinder, which is perfectly shielded and safe. The height of the probe may be adjusted and tightened using the screw. The buttons on the base of the installation is used to vary the number of piezoelectric that may be exposed for each probe assembly.
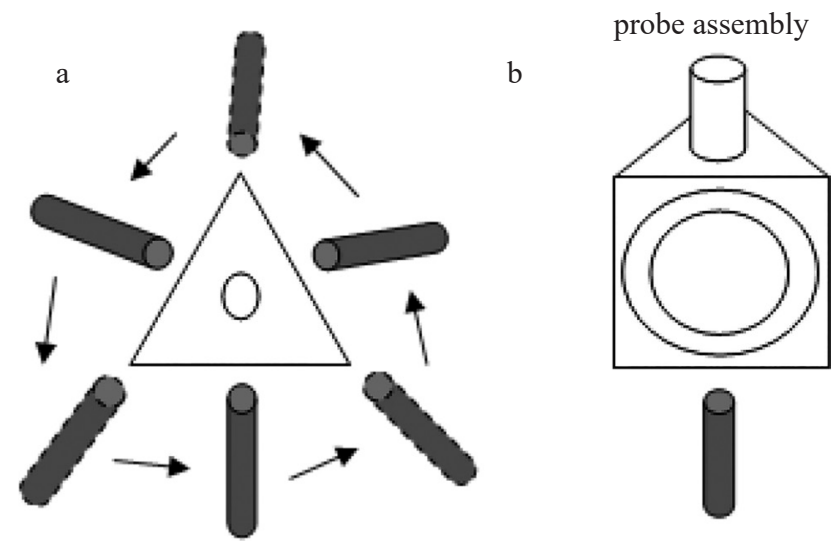

mic condenser

Figure 2: Illustrations of (a) a top view of a mic condenser (dark cylinder) circulating around a probe assembly (triangle with a circle in the middle); (b) the mic condenser is put below the probe assembly.

A validation of the output frequency from the piezoelectric compared to the chosen frequency from the audio generator is conducted using a mic condenser connected to an oscilloscope (GwInstek GOS-630). An illustration is given in Figure 2. For each probe assembly, the mic condenser is put in front of each piezoelectric, one at a time as shown Figure 2(a), and below the probe assembly, shown in Figure 2(b). The result of the signal is shown on the display of the oscilloscope.

The validation test is conducted by assembling the ultrasound generator, i.e., connecting the audio generator, amplifier and probe installation. Next, the audio generator is turned on and set to a certain ultrasound frequency. The mic condenser is positioned according to Figure 2. The output from the piezoelectric may be observed on the display of the oscilloscope (Figure 3). 


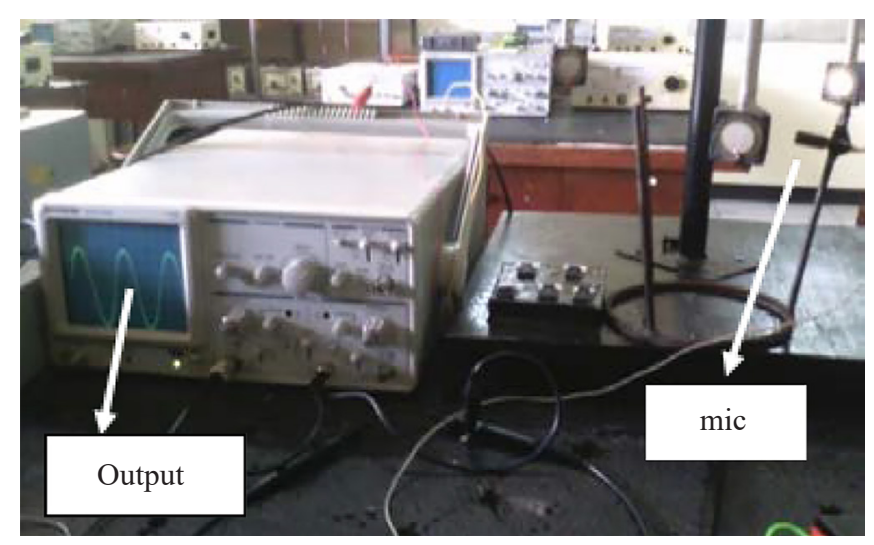

Figure 3: A validation activity of the signal output produced by the piezoelectric compared to the given frequency of the audio generator. The mic condenser (on the right) is placed in front of the piezoelectric. The output signal may be observed by an oscilloscope.

The validation test is conducted for the given frequencies of $25 \mathrm{kHz}, 30 \mathrm{kHz}$ and $35 \mathrm{kHz}$. The result of the test is given in Table 1 . Based on the table, the test confirms that the self-custom-made ultrasound generator is functional, i.e., it produces the expected ultrasonic waves. The sound waves are produced in all directions, that is around (in front of) and below the probes with consistent frequencies. However, the output frequencies from the piezoelectric are a bit higher than the frequencies set on the audio generator. The difference between the actual and intended frequencies is only around $4 \%$ or less and may be caused by beats generated by the three piezoelectric probes. Therefore, we may consider these differences as uncertainty and conclude that the sound generator is ready for use in the exfoliation of graphite layers. The sonication process is done by submerging the probe assembly into a beaker glass containing graphite solution, shown in Figure 4(b).

Table 1: Validation results of the output frequencies of the ultrasound generator.

\begin{tabular}{lcc}
\hline \multirow{2}{*}{$\begin{array}{l}\text { Position of the mic condenser with } \\
\text { respect to the probe assembly }\end{array}$} & \multicolumn{2}{c}{ Frequency $(\mathrm{kHz})$} \\
\cline { 2 - 3 } $\begin{array}{l}\text { Around (in front of) } \\
\text { Below }\end{array}$ & 25 & Audio generator \\
$\begin{array}{l}\text { Around (in front of) } \\
\text { Below }\end{array}$ & 30 & 25.641 \\
$\begin{array}{l}\text { Around (in front of) } \\
\text { Below }\end{array}$ & 35 & 31.250 \\
\hline
\end{tabular}




\section{EXPERIMENTAL}

The equipment needed in this study are as follows: (1) a blender; (2) $200 \mathrm{ml}$ beaker glasses; (3) a digital scale; (4) a cutter; (5) an ultrasound generator, consisting of an audio generator (Figure 4(g)), an amplifier (Figure 4(f)), and a piezoelectric probe installation (Figure 4(h)); (6) an optical microscope (AM 4515 Dinolite); (7) a thermometer; (8) UV-Vis spectrophotometer (Shimadzu UV-Vis 2450); and (9) SEM (JSM 6510 series). On the other hand, the materials used in this study are: (1) $1400 \mathrm{ml}$ of distilled water; (2) $5 \mathrm{~g}$ of graphite powder from the carbon rods of Faber-Castell 2B pencils; and (3) $35 \mathrm{~g}$ of detergent consisting of $20 \%$ linear alkylbenzenesulfonate (LAS).

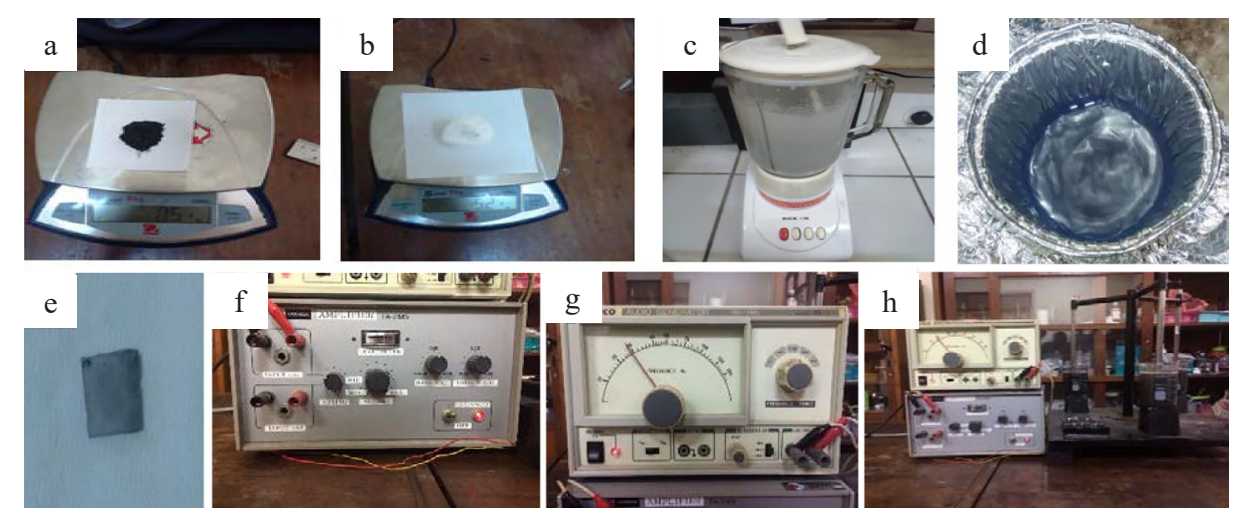

Figure 4: Illustrations of (a) weighing graphite powder; (b) weighing detergent powder; (c) blender for mixing the materials; (d) shallow dish for samples of the solutions after sonication treatment; (e) sample on the glass slide after annealing; (f) Uchida TA-2MS amplifier; (g) CSi/SPECO SS-1 audio generator; and (h) assembly of the self-custom-made ultrasound generator and submerging the probes into the solution.

The experimental phases in the synthesis of GO materials are explained as follows: (1) weighing 5 grams of detergent and 0.5 grams of graphite powder using a digital scale, as shown in Figures 4(a) and 4(b); (2) mixing the materials obtained in step (1) with $200 \mathrm{ml}$ of distilled water using a blender, shown in Figure 4(c); (3) pouring equal amount of the solution into three beaker glasses; (4) placing each beaker glass under a probe assembly; (5) submerging each probe assembly into the beaker glass, shown in Figure 4(h); (6) sonicating the solution for $5 \mathrm{~h}$ with a frequency of $25 \mathrm{kHz}$; (7) measuring the temperatures of the solutions after $1 \mathrm{~h}, 3 \mathrm{~h}$ and $5 \mathrm{~h}$; (8) leaving the solutions to equilibrate for one night; and (9) repeating the above procedures for frequencies of $30 \mathrm{kHz}$ and $35 \mathrm{kHz}$. Next, a similar procedure as above is performed for the variation of the number of piezoelectric probes, i.e., 1,2 and 3 probes, with a constant ultrasound frequency of $30 \mathrm{kHz}$. 
Characterisation of the solutions after the sonication treatments include temperature measurement, optical microscopy, UV-Vis spectroscopy and SEM. Additional treatments are carried out in order that the solutions may be tested using optical microscope and SEM. The samples of the solutions are poured into a shallow dish, as shown in Figure 4(d). A dip coating process is done on one side of a glass slide onto the surface of the solution. The glass slide is then annealed at a temperature of $150^{\circ} \mathrm{C}$ for $20 \mathrm{~min}$, illustrated in Figure 4(e). Subsequently, the sample on the glass slide is placed on running water, and then annealed again with the same temperature and time duration. This cycle is repeated three times.

\section{RESULTS AND DISCUSSION}

Macroscopically, exposing ultrasonic waves from the self-custom-made ultrasound generator towards the solutions cause change of colour and temperature increase of the solutions. The colour of the solution before and after sonication, and also after being left overnight may be observed in Figure 5.

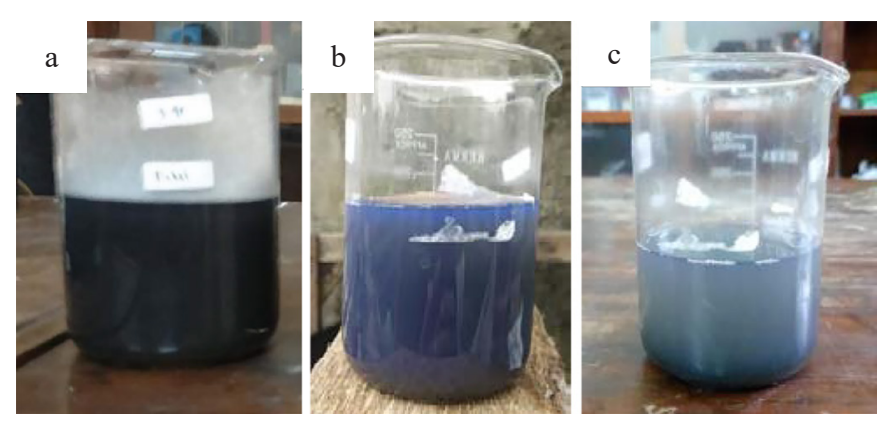

Figure 5: The solution (a) before sonication, (b) after sonication, and (c) after being left for one night.

It can be observed in Figure 5 that colour change took place on the solution after treatments and being left overnight. The initial solution was homogenously dark as it is filled with graphite carbon materials from the $2 \mathrm{~B}$ pencils. After sonication, the solution became blue-ish around the top of the solution and darker around the bottom of the solution. However, the solution turned into greyish colour after being left overnight. Change in the solution colour indicates that exfoliation took place while the sonication process was occurring in the solution. The initial solution was dark as light was absorbed by the graphite solution. As the ultrasound was exposed into the solution, it gradually turned into dark-blue colour that remained until the sonication process was finished. However, after one night the solution equilibrated and reduced to a greyish blue colour. 
The increase in the temperature of the solutions upon sonication may be shown in Table 2. It may be observed that as the number of piezoelectric probes and the frequency were increased, the solution temperatures after $1 \mathrm{~h}, 3 \mathrm{~h}$ and $5 \mathrm{~h}$ of sonication process, i.e., $T_{1}, T_{3}$ and $T_{5}$, respectively, also increased compared to the solution temperature without sonication $\left(T_{0}\right)$, except for $25 \mathrm{kHz}$ ultrasound frequency. For one piezoelectric exposing the solutions, only one degree increase of the temperature was found for $T_{1}, T_{3}$ and $T_{5}$. Obviously, the highest increase of the temperature was obtained for frequency of $35 \mathrm{kHz}$, i.e., reaching $33^{\circ} \mathrm{C}$ for all sonication time durations. The temperature increase of the solution means an increase possibility of graphite layer exfoliation during the sonication process and it may as well contribute to the change of the solution colour.

Table 2: Temperature measurement results of the solution.

\begin{tabular}{lccccc}
\hline \multirow{2}{*}{$\begin{array}{l}\text { Variations } \\
\mathrm{T}_{0}\end{array}$} & \multicolumn{5}{c}{ Solution temperature $\left({ }^{\circ} \mathrm{C}\right)$} \\
\cline { 2 - 6 } & 1 & $\mathrm{~T}_{1}$ & $\mathrm{~T}_{3}$ & $\mathrm{~T}_{5}$ & \\
\hline \multirow{3}{*}{ Number of piezoelectric probes } & 28 & 29 & 29 & 29 \\
& 3 & 28 & 31 & 31 & 31 \\
& 28 & 32 & 32 & 32 \\
\hline \multirow{2}{*}{ Frequency $(\mathrm{kHz})$} & 25 & 28 & 28 & 28 & 28 \\
& 30 & 28 & 32 & 32 & 32 \\
& 35 & 28 & 33 & 33 & 33 \\
\hline
\end{tabular}

Notes: $T_{0}$ is the temperature before sonication; $T_{1}, T_{3}$ and $T_{5}$ are temperatures after $1 \mathrm{~h}, 3 \mathrm{~h}$ and $5 \mathrm{~h}$ of sonication, respectively.

Quantitative evidence in the presence of GO materials in the solution is provided by the UV-Vis results. This may be observed in Figures 6 and 7 for variations of piezoelectric probes and frequency, respectively. The graphs are acquired by subtracting the UV-Vis data of the reference solution (pure surfactant in distilled water) with the UV-Vis data of the GO solutions.

Based on Figure 6, the existence of GO materials in the solutions after sonication may be perceived by an absorbance peak at a wavelength of $273 \mathrm{~nm}$ (red, green and purple arrows). This peak occurs for all number of piezoelectric probes variation. This is a distinct signature of a $\pi \rightarrow \pi^{*}$ transition which signifies double bond carbon atoms $(\mathrm{C}=\mathrm{C})$. The shouldering peaks of the $\mathrm{GO}$ materials are found around $370 \mathrm{~nm}$ signifying $n \rightarrow \pi^{*}$ transitions which show certain functional groups. Increasing the number of piezoelectric probes yields in the lowering of the absorbance values of the $\pi \rightarrow \pi^{*}$ and $n \rightarrow \pi^{*}$ transition peaks. In this case, we may think of increasing the number of piezoelectric probes as increasing the intensity of the ultrasound. Thus, exposing three piezoelectric probes produces the highest intensity of sound and hence the lowest absorbance peaks. 


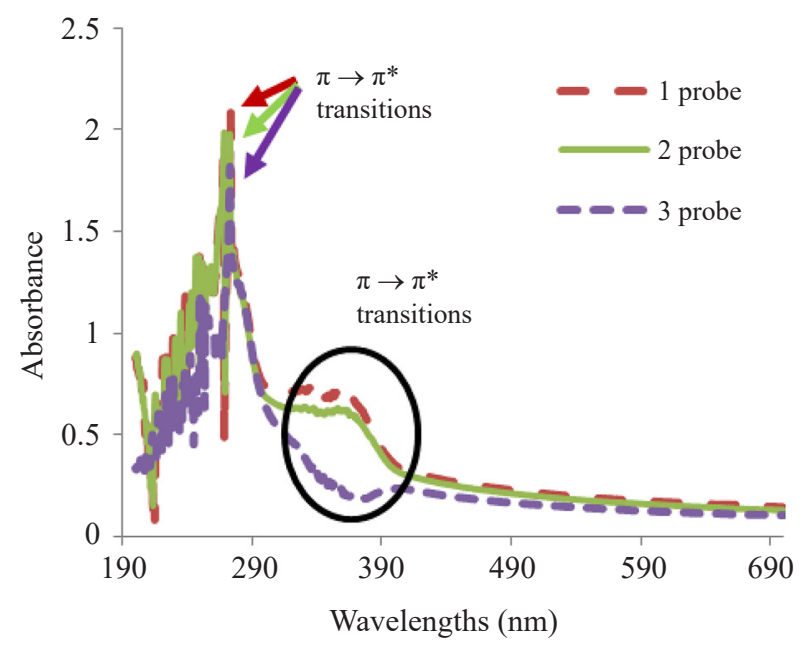

Figure 6: UV-Vis results of GO materials with number of piezoelectric probes variation.

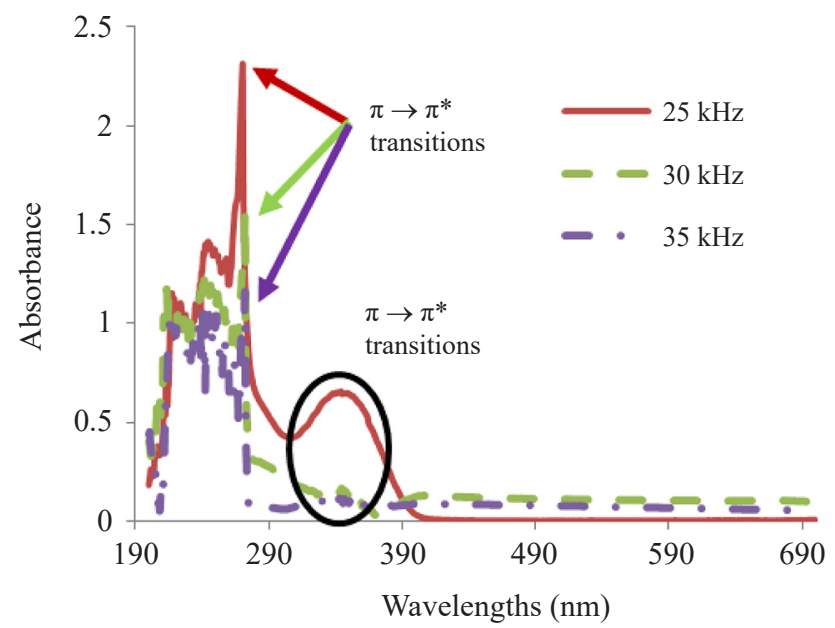

Figure 7: UV-Vis results of GO materials with ultrasound frequency variation.

Similar results are obtained for ultrasound frequency variation (Figure 7). The peaks are obtained at wavelengths of $270 \mathrm{~nm}$ and $350 \mathrm{~nm}$. The former indicates the presence of GO materials, whereas the latter points to functional groups. The absorbance values at the peaks decrease as the ultrasound frequency is increased. The lowest absorbance peak value is obtained at the highest ultrasound frequency of $35 \mathrm{kHz}$. Hence, based on the UV-Vis results, the best performing GO materials produced is by setting the number of piezoelectric probes and frequency of the self-custom-made ultrasound generator to the maximum, i.e., 3 probes and $35 \mathrm{kHz}$, respectively. 
Further verification for the existence of GO materials may be obtained by observing the solidified sample of the solutions using optical microscope and SEM. Optical microscope images of the solidified solutions may be viewed in Figure 8. The images are 500X magnified with the white regions the solidified materials left on the substrate glass. We may discuss the distribution of these white regions, which are affected by different number of piezoelectric probes and ultrasound frequencies. An image of a sample before sonication given in Figure 8(a) shows large bulk of graphite materials on the centre and top left corner of the figure. These bulks of graphite layers indicate that no exfoliation occurs before the sonication process. However, after the sonication process using three piezoelectric probes and $30 \mathrm{kHz}$ ultrasound frequencies, the white regions become relatively smaller and scattered throughout the sample, shown in Figure 8(b). The distribution of the materials is quite homogenous. This shows that the sonication process exfoliates bulk material of graphite layers into smaller pieces. This verifies that the graphite layers are being exfoliated during the sonication process.
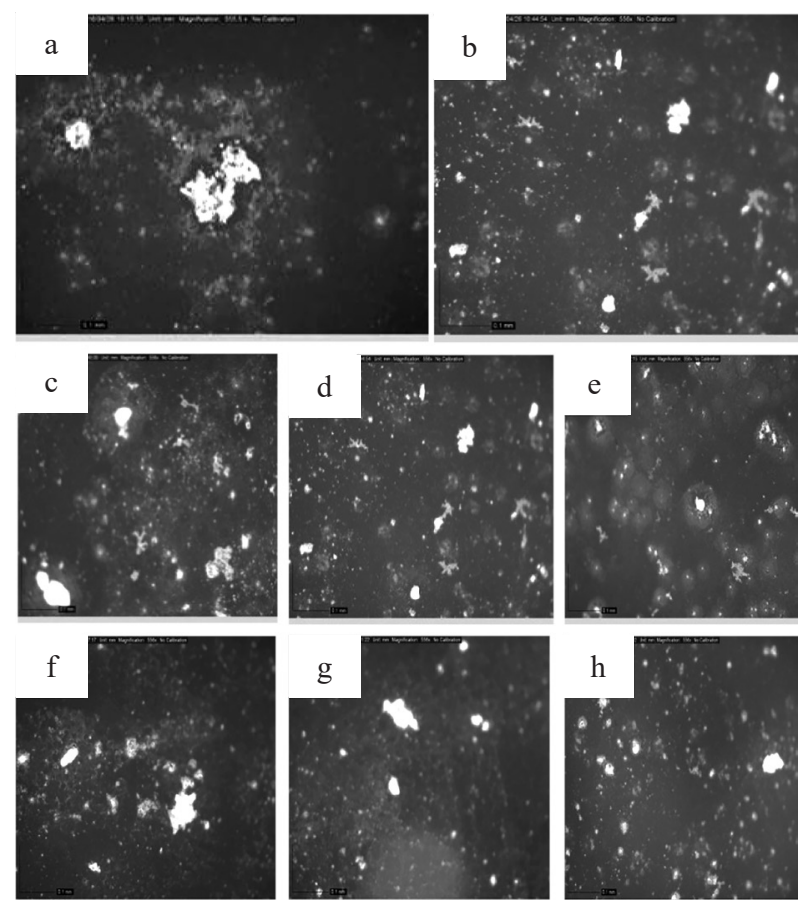

Figure 8: Images of the sample materials through an optical microscope with 500X magnification: (a) before sonication; (b) after sonication with ultrasound frequency of $30 \mathrm{kHz}$ and exposed to 3 piezoelectric probes; (c), (d) and (e) after sonication with 1,2, and 3 probes, respectively; and finally (f), (g) and (h) after sonication with ultrasound frequency of $25 \mathrm{kHz}, 30 \mathrm{kHz}$ and $35 \mathrm{kHz}$, respectively. 
Moreover, we may also look at the effect of the number of probes and ultrasound frequency variation towards the distribution of the sample materials. This is illustrated in Figures 8(c)-8(e) and Figures 8(f)-8(h), respectively. A similar outcome is produced when the number of probes and ultrasound frequency are increased, that is the size of these materials becomes smaller. It may also be observed in Figure 8(h) that the materials are distributed homogenously throughout the sample.
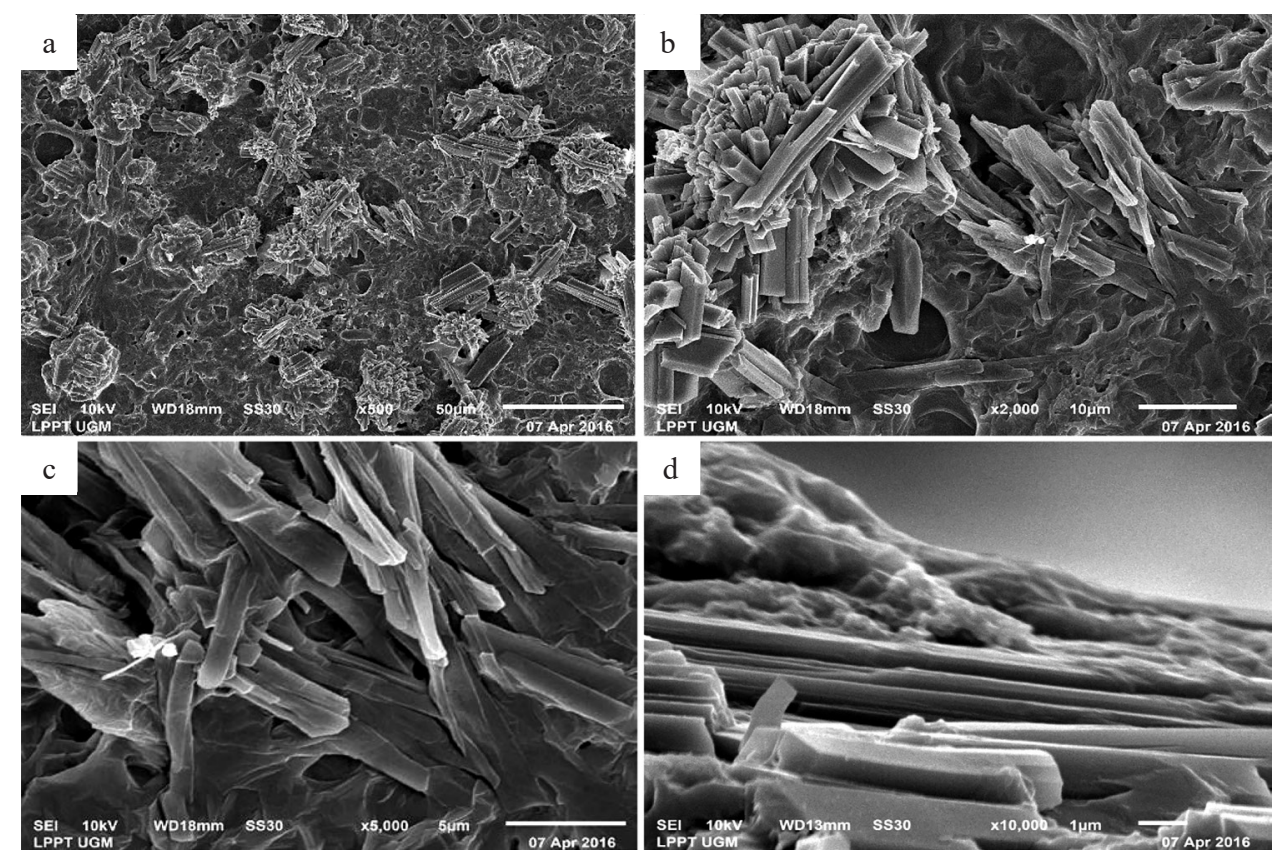

Figure 9: Images of the GO materials obtained from SEM with magnifications of (a) 500X, (b) 2000X, (c) 10000X and (d) 10000X (lateral view).

The surface morphologies of the GO materials are provided by SEM results depicted in Figure 9. The images are obtained from the sample of the solution, which is sonicated with ultrasound frequency of $30 \mathrm{kHz}$ for $5 \mathrm{~h}$ and using 3 piezoelectric probes. The images are obtained with magnifications of 500X, 2000X, 10000X and 10000X (lateral view). Figure 9(a) shows clusters of circular islands throughout the sample and also some rod-like materials. Magnification of 2000X in Figure 9(b) of the sample shows a flower-like structure of broken-rod like materials stacked on top of each other. A rod-like material is obviously seen crossing the flower-like structure. Stacking of materials with layers may be observed in Figure 9(c). The widths of these materials are around 1 to 2 microns. Finally, a lateral image is also provided in Figure 9(d) where layering of materials is perceived with thicknesses of around $300 \mathrm{~nm}$ to 1.5 microns. 


\section{CONCLUSION}

The effects of the number of piezoelectric probes and ultrasound frequency in a selfcustom-made ultrasound generator to exfoliate graphite layers into GO materials have been presented. The best performing GO material is obtained by preparing the ultrasound generator to its maximum settings, i.e., 3 piezoelectric probes and ultrasound frequency of $35 \mathrm{kHz}$. The UV-Vis results show an absorbance peak at around $270 \mathrm{~nm}$, which indicates the presence of GO materials. Moreover, the SEM images show the stacking and layering of GO in the form of broken rod-like materials with widths of 1 to 2 microns and thicknesses of $300 \mathrm{~nm}$ to 1.5 microns. This study is a continuing endeavour of constructing simple and inexpensive devices in order to study novel materials such as GO.

\section{ACKNOWLEDGEMENTS}

The authors would like to thank the Faculty of Mathematics and Natural Sciences, Universitas Negeri Yogyakarta, Indonesia for the funding of this study under the Faculty Grant of Internal Budget Execution List Funding No.1132/UN34.13/ PL/2016. Author Wipsar Sunu also enjoys valuable insights from Bambang Sugeng concerning the English language use while composing this article.

\section{REFERENCES}

1. Takeda, H. et al. (2017). Growth and characterization of strontium-substituted $\mathrm{Ca}_{2} \mathrm{Al}_{2} \mathrm{SiO}_{7}$ piezoelectric single crystals. J. Ceram. Soc. Jap., 125(1), 23-26, https://doi.org/10.2109/jcersj2.16206.

2. Carnacina, I., Larrarte, F. \& Leonardi, N. (2017). Acoustic measurement and morphological features of organic sediment deposits in combined sewer networks. Water Res., 112, 279-290, https://doi.org/10.1016/j.watres. 2017.01.050.

3. He, H. et al. (2017). A flexible self-powered T-ZnO/PVDF/Fabric electricskin with multi-functions of tactile-perception, atmosphere-detection, and self-clean. Nano Energy, 31, 37-48, https://doi.org/10.1016/j.nanoen. 2016.11.020.

4. Yiannakou, M. et al. (2017). MRI-guided coupling for a focused ultrasound system using a top-to-bottom propagation. J. Therap. Ultras., 5(6), 2-8, https://doi.org/10.1186\%2Fs40349-017-0087-x.

5. Ning, H. et al. (2017). Direct patterning of silver electrodes with $2.4 \mu \mathrm{m}$ channel length by piezoelectric inkjet printing. J. Coll. Interf. Sci., 487, 6872, https://doi.org/10.1016/j.jcis.2016.10.016. 
6. Wang, Z. L. \& Song, J. (2006). Piezoelectric nanogenerators based on zinc oxide nanowires arrays. Sci., 312, 242-246, https://doi.org/10.1126/ science. 1124005.

7. Laurenti, M. et al. (2017). Lead-free piezoelectrics: $\mathrm{V}^{3+}$ to $\mathrm{V}^{5+}$ ion conversion promoting the performances of V-doped zinc oxide. Sci. Rep., 7(41957), 1-13, https://doi.org/10.1038/srep41957.

8. Prabahar, K. et al. (2017). Effect of deposition temperature on the microstructure, ferroelectric, and mechanical properties of lead free BCZT ceramics thin films. Ceram. Int., 43(6), 5356-5361, https://doi.org/ 10.1016\%2Fj.ceramint.2017.01.032.

9. Miao, H. et al. (2017). A new omnidirectional shear horizontal wave transducer using face-shear $\left(\mathrm{d}_{24}\right)$ piezoelectric ring array. Ultrason., 74, 167-173, https://doi.org/10.1016/j.ultras.2016.10.011.

10. Woodard, S. E. (2005). Methods to increase sound fidelity and quality produced from piezoelectric devices. J. Sound Vib., 280, 127-140, https://doi.org/10.1016/j.jsv.2003.12.039.

11. Wang, S., Yi, M. \& Shen, Z. (2016). The effect of surfactants and their concentrations on the liquid exfoliation of graphene. RSC Adv, 6, 5670556710, https://doi.org/10.1039/C6RA10933K.

12. Hernandez, Y. et al. (2008). High yield production of graphene by liquidphase exfoliation of graphite. Nat. Nanotechnol., 3, 563-568, https://doi. org/10.1038/nnano.2008.215.

13. Khan, U. et al. (2010). High-concentration solvent exfoliation of graphene. Small, 6, 864-871, https://doi.org/10.1002/smll.200902066.

14. Bang, J. H. \& Sulick, K. S. (2010). Applications of ultrasound to the synthesis of nanostructured materials. Adv. Mater., 22, 1039-1059, https:// doi.org/10.1002/adma.200904093.

15. Durge, R., Kshirsagar, R. V. \& Tambe, P. (2014). Effect of sonication energy on the yield of graphene nanosheet by liquid-phase exfoliation of graphene. Proc. Eng., 97, 1457-1465, https://doi.org/10.1016/j.proeng.2014.12.429.

16. Yi, M. \& Shen, Z. (2014). Kitchen blender for producing high-quality few-layer graphene. Carbon, 78, 622-626, https://doi.org/10.1016/j.carbon. 2014.07.035.

17. Tang, L., Li, X., Du, D. \& He, C. (2012). Fabrication of multilayer films from regenerated cellulose and graphene oxide through layer-by-layer assembly. Progr. Nat. Sci. Mater. Int., 22, 341-346, https://doi.org/10.1016/ j.pnsc.2012.06.005.

18. Reina, A. et al. (2009). Large area, few-layer graphene films on arbitrary substrates by chemical vapor deposition. Nano Lett., 9, 30-35, https://doi. org $/ 10.1021 / \mathrm{n} 1801827 \mathrm{v}$. 
19. Marcano, D. C. et al. (2010). Improved synthesis of graphene oxide. ACS Nano, 4, 4806-4814, https://doi.org/10.1021/nn1006368.

20. Obraztsov, A. N. (2009). Chemical vapour deposition: Making graphene on a large scale. Nat. Nanotechnol., 4, 212-213, https://doi.org/10.1038/ nnano.2009.67.

21. Chen, Z. et al. (2011). Three-dimensional flexible and conductive interconnected graphene networks grown by chemical vapour deposition. Nat. Mater., 10, 424-428, https://doi.org/10.1038/nmat3001.

22. Sutter, P. W., Flege, J. I. \& Sutter, E. A. (2008). Epitaxial graphene on ruthenium. Nat. Mater., 7, 406-411, https://doi.org/10.1038/nmat2166.

23. Yang, W. et al. (2013). Epitaxial growth of single-domain graphene on hexagonal boron nitride. Nat. Mater., 12, 792-797, https://doi.org/10.1038/ nmat3695.

24. Stankovich, S. et al. (2007). Synthesis of graphene based nanosheets via chemical reduction of exfoliated graphite oxide. Carbon, 45, 1558-1565, https://doi.org/10.1016/j.carbon.2007.02.034.

25. Pei, S. \& Cheng, H. M. (2012). The reduction of graphene oxide. Carbon, 50, 3210-3228, https://doi.org/10.1016/j.carbon.2011.11.010.

26. Yi, M. \& Shen, Z. (2015). A review on mechanical exfoliation for the scalable production of graphene. J. Mater. Chem. A, 3, 11700-11715, https://doi.org/10.1039/C5TA00252D.

27. Yi, M., Shen, Z. \& Zhu, J. (2014). A fluid dynamics route for producing graphene and its analogues. Chin. Sci. Bull., 59(16), 1794-1799, https://doi. org/10.1007/s11434-014-0303-9.

28. Yi, M. \& Shen, Z. (2016). Fluid dynamics: An emerging route for the scalable production of graphene in the last five years. RSC Adv., 6, 72525-72536, https://doi.org/10.1039/C6RA15269D.

29. Fikri, A. A. et al. (2016). Synthesis of graphene oxide via liquid exfoliation using self-custom-made tweeter piezoelectric ultrasound generator and assisted by surfactant from commercial detergent. Am. J. Appl. Sci., 13(11), 1129-1135, https://doi.org/10.3844/ajassp.2016.1129.1135.

30. Murat, A. et al. (2012). The synthesis of graphene sheets with controlled thickness and order using surfactant assisted electrochemical processes. Carbon, 50, 142-152, https://doi.org/10.1016/j.carbon.2011.07.064.

31. Park, S. \& Rouff, R. S. (2009). Chemical methods for the production of graphenes. Nat. Nanotechnol., 4, 217-224, https://doi.org/10.1038/ nnano.2009.58.

32. Li, D., Müller, M. B. \& Gilje, S. (2008). Processable aqueous dispersions of graphene nanosheets. Nat. Nanotechnol., 3, 101-105, https://doi. org/10.1038/nnano.2007.451. 
33. Terrones, M. et al. (2010). Graphene and graphite nanoribbons: Morphology, properties, synthesis, defects and applications. Nano Today, 5, 351-372, https://doi.org/10.1016/j.nantod.2010.06.010.

34. Shao, Y. et al. (2010). Graphene based electrochemical sensors and biosensors: A review. Electroanal., 22, 1027-1036, https://doi.org/10.1002/ elan.200900571.

35. Liu, Y. et al. (2010). Biocompatible graphene-oxide based glucose biosensors. Langmuir, 26, 6158-6160. https://doi.org/10.1021/la100886x.

36. Wang, L. et al. (2009). Graphene oxide as an ideal substrate for hydrogen storage. ACS Nano, 3, 2995-3000, https://doi.org/10.1021/nn900667s.

37. Tylianakis, E., Psofogiannakis, G. M. \& Froudakis, G. E. (2010). Lidoped pillared graphene-oxide: A graphene based nanostructured material for hydrogen storage. J. Phys. Chem. Lett., 1, 2459-2464, https://doi.org/ 10.1021/jz100733z.

38. Kim, B. H. et al. (2012). Thermally modulated multi layered graphene oxide for hydrogen storage. Phys. Chem. Chem. Phys., 14, 1480-1484, https://doi.org/10.1039/C2CP23683D.

39. Joshi, R. K. et al. (2014). Precise and ultrafast molecular sieving through graphene oxide membranes. Sci., 343, 752-754, https://doi.org/10.1126/ science. 1245711.

40. $\mathrm{Xu}, \mathrm{C}$. et al. (2013). Graphene oxide $\mathrm{TiO}_{2}$ composite filtration membranes and their potential application for water purification. Carbon, 62, 465-471, https://doi.org/10.3390/membranes6040057.

41. Chung, C. et al. (2013). Biomedical applications of graphene and graphene Oxide. Acc. Chem. Res., 46, 2211-2224, https://doi.org/10.1021/ar300159f.

42. Loh, K. P. et al. (2010). Graphene oxide as a chemically tunable platform for optical applications. Nat. Chem., 2, 1015-1024, https://doi.org/10.1038/ nchem.907.

43. Zhang, D., Liu, X. \& Wang, X. (2011). Green synthesis of graphene oxide sheets decorated by silver nanoprisms and their anti-bacterial properties. J. Inorg. Biochem., 105, 1181-1186, https://doi.org/10.1016/j. jinorgbio.2011.05.014.

44. Tang, J. et al. (2013). Graphene-oxide silver nanocomposite as a highly effective antibacterial agent with species specific mechanism. ACS Appl. Mater. Interf., 5, 3867-3874, https://doi.org/10.1021/am4005495.

45. Shao, W. et al. (2015). Anti-bacterial performances and biocompatibility of bacterial cellulose/graphene oxide composites. RSC Adv., 5, 4795-4803, https://doi.org/10.1039/C4RA13057J. 